\section{Practice Guideline}

Check for updates

\section{OPEN ACCESS}

Received: Dec 18, 2018

Revised: Dec 27, 2018

Accepted: Dec 27, 2018

Correspondence to

Jae-Kwan Lee

Department of Obstetrics and Gynecology,

Korea University Medical Center, Korea

University College of Medicine,

148 Gurodong-ro, Guro-gu, Seoul 08308,

Korea.

E-mail: jklee38@gmail.com

Copyright $\odot$ 2019. Asian Society of Gynecologic Oncology, Korean Society of Gynecologic Oncology

This is an Open Access article distributed under the terms of the Creative Commons Attribution Non-Commercial License (https:// creativecommons.org/licenses/by-nc/4.0/) which permits unrestricted non-commercial use, distribution, and reproduction in any medium, provided the original work is properly cited.

ORCID IDS

Kyung-Jin Min (iD)

https://orcid.org/0000-0002-5783-4968 Sang-Hoon Kwon (iD

https://orcid.org/0000-0002-9121-3954 Kidong Kim (D)

https://orcid.org/0000-0001-9254-6024 Sunghoon Kim (iD)

https://orcid.org/0000-0002-1645-7473

Hyun Jung Kim (iD)

https://orcid.org/0000-0003-2018-2385 Seok Ju Seong (i)

https://orcid.org/0000-0003-3820-3412

Yong Jung Song (i)

https://orcid.org/0000-0002-6103-2466

\title{
Clinical guideline for 9-valent HPV vaccine: Korean Society of Gynecologic Oncology Guideline
}

\author{
Kyung-Jin Min (1), ${ }^{1}$ Sang-Hoon Kwon (1), ${ }^{2}$ Kidong Kim $(1),{ }^{3}$ Sunghoon Kim (1), ${ }^{4}$ \\ Hyun Jung Kim $\left(\mathbb{D},{ }^{5}\right.$ Seok Ju Seong $\mathbb{( \mathbb { D } ,},{ }^{6}$ Yong Jung Song $\mathbb{( \mathbb { D } ,},{ }^{7}$ Keun Ho Lee $\mathbb{D},{ }^{8}$

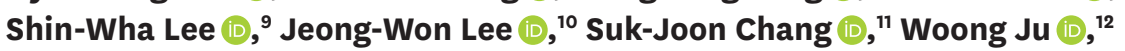 \\ Young-Tak Kim $\mathbb{D}^{,}, 9$ Jae-Kwan Lee $\mathbb{1}^{1}$
}

'Department of Obstetrics and Gynecology, Korea University Medical Center, Korea University College of Medicine, Seoul, Korea

${ }^{2}$ Department of Obstetrics and Gynecology, Keimyung University School of Medicine, Daegu, Korea ${ }^{3}$ Department of Obstetrics and Gynecology, Seoul National University Bundang Hospital, Seongnam, Korea ${ }^{4}$ Department of Obstetrics and Gynecology, Yonsei University College of Medicine, Seoul, Korea ${ }^{5}$ Department of Preventive Medicine, Korea University College of Medicine, Seoul, Korea ${ }^{6}$ Department of Obstetrics and Gynecology, CHA Gangnam Medical Center, CHA University, Seoul, Korea ${ }^{7}$ Department of Obstetrics and Gynecology, Pusan National University School of Medicine, Yangsan, Korea ${ }^{8}$ Department of Obstetrics and Gynecology, Seoul St. Mary's Hospital, College of Medicine, The Catholic University of Korea, Seoul, Korea

${ }^{9}$ Department of Obstetrics and Gynecology, Asan Medical center, University of Ulsan College of Medicine, Seoul, Korea

${ }^{10}$ Department of Obstetrics and Gynecology, Samsung Medical Center, Sungkyunkwan University School of Medicine, Seoul, Korea

"Division of Gynecologic Oncology, Department of Obstetrics and Gynecology, Ajou University School of Medicine, Suwon, Korea

${ }^{12}$ Department of Obstetrics and Gynecology, Ewha Womans University Mokdong Hospital, Ewha Womans University School of Medicine, Seoul, Korea

\section{ABSTRACT}

In 2016, 9-valent human papillomavirus (HPV) vaccine has been newly introduced in Korea, thus the need to develop recommendations for the vaccine has raised. Until we decide to develop a guideline, no further studies on the bi-valent or quadri-valent HPV vaccine have been announced. We searched and reviewed the literatures focused on the efficacy of 9-valent HPV vaccine, the ideal age of 3-dose schedule vaccination, the efficacy of 9-valent HPV vaccine in middle-aged women, the efficacy of the 2-dose schedule vaccination, the safety of 9-valent HPV vaccine, the possibility of additional 9-valent HPV vaccination, and crossvaccination of 9-valent HPV vaccine. So, Korean Society of Gynecologic Oncology (KSGO) developed a guideline only for 9-valent HPV vaccine.

Keywords: Papillomavirus Vaccines; Practice Guidelines; Female; Male 
Keun Ho Lee (iD

https://orcid.org/0000-0001-9005-7796 Shin-Wha Lee (iD

https://orcid.org/0000-0002-5088-1905 Jeong-Won Lee iD

https://orcid.org/0000-0002-6945-0398 suk-Joon Chang (iD)

https://orcid.org/0000-0002-0558-0038 woong Ju iD

https://orcid.org/0000-0002-0013-8304

Young-Tak Kim (iD)

https://orcid.org/0000-0002-2126-8503

Jae-Kwan Lee (iD

https://orcid.org/0000-0003-3101-6403

Conflict of Interest

No potential conflict of interest relevant to this article was reported.

Author Contributions

Data curation: M.K.J., K.S.H., K.K., K.S., K.H.J., S.S.J., S.Y.J., L.K.H., L.S.H., L.J.W., C.S.J., J.W., K.Y.T., L.J.K.; Formal analysis: M.K.J., K.S.H., K.K., K.S., K.H.J., S.S.J., S.Y.J., L.K.H., L.S.H., L.J.W., C.S.J., J.W., K.Y.T., L.J.K.; Investigation: M.K.J., K.S.H., K.K., K.S., K.H.J., S.S.J., S.Y.J., L.K.H., L.S.H., L.J.W., C.S.J., J.W., K.Y.T., L.J.K.; Methodology: K.H.J.; Project administration: L.J.K.; Software: K.H.J.; Writing - original draft: M.K.J., K.S.H., K.K., K.S., K.H.J., S.S.J., S.Y.J., L.K.H., L.S.H., L.J.W., C.S.J., J.W., K.Y.T., L.J.K.; Writing - review \& editing: M.K.J., K.S.H., K.K., K.S., K.H.J., S.S.J., S.Y.J., L.K.H., L.S.H., L.J.W., C.S.J., J.W., K.Y.T., L.J.K.

\section{Key question 1: is the 9-valent HPV vaccine effective?}

- The 9-valent HPV vaccine is effective in preventing the diseases related to $9 \mathrm{HPV}$ types (HPV 6, 11, 16, 18, 31, 33, 45, 52, and 58) (1A).

\section{Key question 2: what is the recommended age for the 3-dose schedule of} 9-valent HPV vaccine?

- The best age for the 3-dose schedule of 9-valent HPV vaccine is between 9 and 26 years in men and women (1A).

3. Key question 3: is the 9-valent HPV vaccine effective in middle-aged women?

- The 9-valent HPV vaccination in middle-aged women (27-45 years) has insufficient evidence for preventive effects, but can be clinically determined considering the circumstances of the subjects (2E).

4. Key question 4: is the 2-dose schedule of 9-valent HPV vaccine effective?

- The 2-dose schedule of 9-valent HPV vaccine is effective for men and women aged 9-14 years (1A).

\section{Key question 5: is the 9-valent HPV vaccine safe?}

- The 9-valent HPV vaccine is as safe as other HPV vaccines (1A).

- For pregnant women, the 9-valent HPV vaccination is not recommended (1E).

- Breastfeeding women can be vaccinated with 9-valent HPV vaccine (1E).

- The 9-valent HPV vaccine can be co-administered with the meningococcal conjugate vaccine or tetanus, diphtheria, and acellular pertussis (Tdap) Vaccine (1A).

\section{Key question 6: is it possible to complete vaccination with the 9-valent} HPV vaccine for women previously inoculated with the other types of HPV vaccines?

- In cases of vaccinations started with other types of HPV vaccines, the 9-valent HPV vaccine may be used to continue or complete the vaccination ( ${ }^{\star}$ limited to cases where the previous vaccine types are unknown or unavailable) (E).

\section{Key question 7: is an additional 9-valent HPV vaccination possible to women} who have already completed a series with another HPV vaccine product?

- For women aged 12-26 years who have completed the quadri-valent HPV vaccination, an additional 9-valent HPV vaccination can be clinically determined considering the circumstances of the subject (2B).

\section{INTRODUCTION}

Human papillomavirus (HPV) can often be infected in sexually active women [1]. About 40 genotypes of HPV can infect the female reproductive system, of which $15 \mathrm{HPV}$ types are referred to as a carcinogenic or high-risk virus due to their association with gynecologic cancers, including cervical cancer [2]. Most infections are known to be extinguished within 2 years, and the major cause of cervical cancer and pre-cancer lesions is a persistent infection caused by highrisk HPV for more than 2 years [2]. Among these, HPV 16 and 18 are the 2 most prevalent types, 
which cause about $70 \%$ of invasive cervical cancer worldwide, accounting for $50 \%$ of moderate and severe cervical dysplasia [3,4]. Other types, including HPV 31, 33, 45, 52, and 58, account for about 90 percent of all invasive cervical cancer. In addition to cervical cancer, HPV 16 and 18 cause $40 \%$ to $50 \%$ of invasive vulvar cancer and 70 percent of vaginal cancer. After the Korean Society of Gynecologic Oncology revised the clinical recommendation for preventive vaccination of cervical cancer in 2011 and 2016 [5-7], no further studies on the bi-valent or quadri-valent HPV vaccine have been announced. However, 9-valent HPV vaccine has been newly introduced in Korea, thus the need to develop recommendations for the vaccine has raised.

\section{MECHANISMS OF ACTION OF THE 9-VALENT HPV VACCINE}

To counteract the disease prevention capability of the 2 existing preventive vaccines, which provided only the preventive effects about HPV16- and 18-related precancerous lesions and malignant tumors, the 9-valent HPV vaccine was manufactured by adding HPV 31, 33, 45, 52, and $58 \mathrm{~L} 1$ virus-like parts (VLPs) in addition to HPV16 and 18 [8]. After 3 phase II studies were conducted with 9 HPV type L1 VLPs as an experimental group and quadri-valent HPV vaccine as a control group, the efficacy, effectiveness, and safety of the 9-valent HPV vaccine were identified. The final 0.5-mLof the 9-valent vaccine contains approximately $30 \mathrm{mcg}$ of HPV type $6 \mathrm{~L} 1$ protein, $40 \mathrm{mcg}$ of HPV type $11 \mathrm{~L} 1$ protein, $60 \mathrm{mcg}$ of HPV type $16 \mathrm{~L} 1$ protein, 40 mcg of HPV type $18 \mathrm{~L} 1$ protein, $20 \mathrm{mcg}$ of HPV type $31 \mathrm{~L} 1$ protein, $20 \mathrm{mcg}$ of HPV type $33 \mathrm{~L} 1$ protein, $20 \mathrm{mcg}$ of HPV type $45 \mathrm{~L} 1$ protein, $20 \mathrm{mcg}$ of HPV type $52 \mathrm{~L} 1$ protein, and $20 \mathrm{mcg}$ of HPV type 58 L1 protein.

\section{EVIDENCE OF 9-VALENT HPV VACCINE}

The process of developing clinical guideline and the evidence table for each Key Questions are described in Supplementary Materials (Supplements 1 and 2).

\section{Key question 1: is the 9-valent HPV vaccine effective?}

Joura et al. [9] conducted a randomized, international, multi-center, double-blind, and phase 2b-3 study to check the efficacy and immunogenicity of the 9-valent HPV vaccine. Since quadri-valent HPV vaccine was proven effective, a total of 14,215 women aged 16-26 years was included in this study for the comparison between the quadri-valent and 9-valent HPV vaccine. The study found that the immunogenicity for HPV 6, 11, 16, and 18 was similar between the 2 groups, and geometric mean titers (GMTs) remained similar as well about 2 years after the vaccination. The vaccine efficacy for HPV 31, 33, 45, 51, and 58 was superior with the results of $97.4 \%$ (95\% confidence interval $[\mathrm{CI}]=85.0-99.9)$ for uterine cervix/vulva/ vaginal disease, $97.1 \%$ (95\% CI=83.5-99.9) for cervical intraepithelial neoplasm 2/3 (CIN 2/3) or adenocarcinoma in situ, 100\% (95\% CI=39.4-100) for CIN3, 96.0\% (95\% CI=94.6-97.1) for persistent infection for more than 6 months, $96.7 \%$ (95\% CI=95.1-97.9) for persistent infection for more than 12 months, 90.2\% (95\% CI=75.0-96.8) for cervical procedure including punch biopsy, and $92.9 \%(95 \% \mathrm{CI}=90.2-95.1)$ for abnormal cervical cytology.

The researchers, including Van Damme, conducted a study in boys and girls aged 9-15 years to confirm that the immune response was non-superior than young women's [10]. This study included 400 women between the ages of 16 and 26 as a control group, and 600 boys and 
1,800 girls aged 9-15 for an experimental group. Non-superiority was defined as the ratio of 2-sided 95\% CI for each HPV type greater than 0.67. GMTs for all types of HPVs increased for all subjects, and young boys and girls were found to be non-superior than young women. The antibodies to the HPV type were identified up to 2.5 years after the third dose.

In 2015, Castellsagué et al. [11] demonstrated the efficacy of the 9-valent HPV vaccine in men. This study was judged to be observational because no randomization process was mentioned. The study selected 16-26-year-old women as a control group and 1,106 self-identified as heterosexual men (HM) and 313 self-identified as men having sex with men (MSM) of similar age as an experimental group. Anti-HPV GMTs at month 7 among 16-26-year-old HM were non-inferior to anti-HPV GMTs among 16-26-year-old girls and women. The GMT ratios for MSM relative to HM were generally similar to those previously observed in clinical trials with quadri-valent HPV vaccine. Across all clinical trials, at least $99.5 \%$ of individuals included in the analyses for each of the 9 vaccine HPV types became seropositive by month 7 .

Van Damme et al. [12] identified the immunogenicity and positivity ratio of antibody by conducting a randomized controlled study to confirm the efficacy of the 9-valent HPV vaccine in men. At 7-months after the vaccination, GMTs for HPV 6, 11, 16, and 18 were similar to the quadri-valent HPV vaccination group, yet superior for HPV 31, 33, 45, 52, and 58 . The positivity ratio of antibody was also observed as $100 \%$ for $9 \mathrm{HPV}$ types when the 9 -valent HPV was inoculated.

Based on the results of 3 randomization studies and 1 observational study, the level of evidence for the effect of 9-valent HPV vaccine was determined to be HIGH. The above results determined that the recommendations:

- The 9-valent HPV vaccine is effective in preventing the diseases related to 9 HPV types (HPV 6, 11, 16, 18, 31, 33, 45, 52, and 58) (1A).

\section{Key question 2: what is the recommended age for the 3-dose schedule of 9-valent HPV vaccine?}

In 4 studies selected as evidence in key question 1, the efficacy of 9-valent HPV vaccine was checked in men and women aged 9-26 for a total 3 dose scheduled 0, 2, and 6 months. As the efficacy of the 9-valent HPV vaccine was identified in key question 1 from 3 randomization studies and 1 observational study, the recommendation of this key question was decided as follows:

- The best age for the 3-dose schedule of 9-valent HPV vaccine is between 9 and 26 years in men and women $(1 \mathrm{~A})$.

3. Key question 3: is the 9-valent HPV vaccine effective in middle-aged women? Researches associated with 9-valent HPV vaccine in middle-aged women over age 27 was not published until the articles were searched for writing recommendations. However, 9-valent HPV vaccine was tested for efficacy compared to the quadri-valent HPV vaccine which identified efficacy in middle-aged women in 3 studies [13-15]. Based on these findings, the 
European Union allowed the 9-valent HPV vaccination in men and women over the age of 9 because they decided that 9-valent HPV vaccine could benefit middle-aged women between the ages of 27-45. In Canada, 9-valent HPV vaccination was authorized for women aged 9-45 and men aged 9-26 because 9-valent HPV vaccine could have similar safety profiles and effects in middle-aged women between 27-45 year old after confirming the efficacy and safety of the 9-valent HPV vaccine in 16-26 year old women and the quadri-valent HPV vaccine in 16-45 year old women.

With no study result on this key question, the recommendation level was weak and the evidence level was determined to be $\mathrm{E}$ by selecting the judgement from other countries as a reference. The above results determined that the recommendations.

- The 9-valent HPV vaccine can be inoculated in middle-aged women (27-45 years old) by taking into account clinical judgment of individual risk and situations, although there is insufficient evidence for preventive effects (2E).

\section{Key question 4: is the 2-dose schedule of 9-valent HPV vaccine effective?} Only 1 randomized study conducted by Iversen et al. [16] was found to support this key question. This was conducted as a randomized, multi-centered, and open label study to ensure that 2 dose vaccination of 9-valent HPV vaccine in 9-14 year old boys and girls is noninferior to the 3 dose vaccination in 9-26 years old women. In general, HPV test or cervical cytology should be carried out directly at the uterine cervix in order to check the effectiveness of vaccine. However, the effectiveness of the vaccine was indirectly confirmed through antibody titers in this case of young adolescent since they had little possibility of exposure to HPV. Similar studies conducted in the bi-valent and quadri-valent vaccine were adopted as the evidence to authorize 2 dose vaccination by WHO and other agencies. For this reason, the study by Iversen et al. [16] was accepted as an evidence of this key question although it is the only 1 randomized study.

An experimental group of the study with the 9-valent HPV vaccination included adolescents aged 9-14 years who were sexually naïve at enrollment, and the control group included the women between the ages of 9 and 26 who had no more than 4 sexual partners and did not have abnormal cervical cytology or other cervical disorders. In order to identify the efficacy of 2 dose vaccination in 9-14 year old adolescent, the 9-valent HPV vaccine was inoculated with 2 dose to 9-14 aged girls ( 0 and 6 months), 2 dose to $9-14$ aged man ( 0 and 6 months), 2 dose to 9-14 aged adolescent ( 0 and 12 months), 3 dose to $9-14$ aged girls ( 0,2 , and 6 months), and 3 dose to $16-26$ aged woman $(0,2$, and 6 months).

Antibody titer in 2 dose vaccinated groups was non-inferior compared to that of 3 dose vaccinated groups in HPV antibody assay conducted 4 weeks after the last vaccination. In addition, antibody titer for 8 HPV types was observed higher, with the exception of HPV 45, in 2 dose vaccinated group at 0 and 12 months compared to the 0 and 6 months-vaccinated group.

Since the evidence of 2 dose vaccination with the 9-valent HPV vaccine was interpreted in a randomized study, the level of evidence was determined to be HIGH, and the level of recommendation was strong. The above results determined the following recommendations: 
- The 2-dose schedule of 9-valent HPV vaccine is effective for men and women aged 9-14 years (1A).

\section{Key question 5: is the 9-valent HPV vaccine safe?}

This key question identified the adverse events of 9-valent HPV vaccine, adverse events in pregnant or breastfeeding women, and the possibility of simultaneous inoculation with other vaccines.

First of all, the side effects of the 9-valent vaccine were identified through 6 studies. Side effects identified in the study by Joura et al. [9] are as follows. Pain, edema, rash, and itching sensation at inoculation site were the most common side effect, while systemic side effects included headaches, fever, nausea, dizziness, and fatigue. However, these had been similarly observed in quadri-valent vaccination groups, and it was very rare for both groups to stop vaccination because of the side effects. Van Damme et al. [10] found side effects in boys and girls aged 9-15 years. Similar to the control group of 16-26 aged woman, side effects at inoculation site such as pain, edema, rash and itching sense were most commonly observed. However, less pain and edema were observed in boy than in women. Headache, fever, and fatigue which were the most common systemic side effects were observed at a similar rate in adolescent and young aged women. In the studies by Castellsagué et al. [11] and Van Damme et al., [12] men aged 16-26 years experienced similar side effects as woman had in the previous studies, but with less percentage. Vesikari et al. [17] confirmed the safety of 9-valent vaccine in girls aged 9-15 years. Even though swelling at inoculation site was observed more in 9-valent vaccinated group ( $47.8 \%$ vs. $36.0 \%$; $p=0.003$ ), the overall safety was similar when compared to the quadri-valent vaccinated group. The integrated analysis of 7 phase 3 studies on 9-valent HPV vaccine by Moreira et al. [18] demonstrated that the cases with 1 or more side effects were $92.7 \%$ in women and $82.5 \%$ in men. Typical side effects included pain, edema, rash, and itching sense at inoculation site, headaches, fever, nausea, dizziness, and fatigue as a vaccine-related systemic effects, and severe vaccination-related side effects were not detected in both men and women.

Moreira et al. [18] confirmed the results of pregnancy among those who participated as pregnant women in the study by Joura et al. [9] Normal birth rates were $77.3 \%$ in 9-valent HPV vaccinated group and $76.7 \%$ in quadri-valent vaccinated group, respectively, while abnormal pregnancy rates were $2.0 \%$ and $2.2 \%$ in each group. The miscarriage rates were $19.9 \%$ and $20.8 \%$, and the ratio of ectopic pregnancy and spontaneous abortion was similar to that of quadri-valent vaccine group and general pregnant women. This confirms that the 9-valent vaccine is harmless to pregnant women, but, it is not recommended for pregnant women to receive 9-valent HPV vaccine since no clinical research has been conducted on pregnant women. In addition, the study included 72 breastfeeding women, and no adverse effects were found with 9-valent HPV vaccination [18]. This study was judged to be a cohort study because it was an integrated analysis based on the randomized trials.

Schilling et al. [19] and Kosalaraksa et al. [20] confirmed that the simultaneous administration of the 9-valent HPV vaccine with meningococcal vaccine, diphtheria, tetanus, diphtheria, and acellular pertussis (Tdap) vaccine, or poliomyelitis vaccine showed similar antibody titer and side effects between 2 groups $[19,20]$. 
Based on 4 randomized studies and 2 observational studies, the levels of evidence and recommendation were classified as HIGH and strong for the safety of 9-valent HPV vaccine. Although 9-valent HPV vaccine may be safe for breastfeeding women since L1 VLPs were used, the levels of evidence and recommendation were classified as $\mathrm{E}$ and strong, respectively. The levels of evidence and recommendation for the simultaneous vaccination along with other vaccines were decided as HIGH and STRONG based on the randomized studies.

The above results determined the following recommendations:

- The 9-valent HPV vaccine is as safe as other HPV vaccines (1A).

- For pregnant women, the 9-valent HPV vaccination is not recommended (1E).

- Breastfeeding women can be vaccinated with 9-valent HPV vaccine (1E).

- The 9-valent HPV vaccine can be co-administered with the meningococcal conjugate vaccine or Tdap vaccine (1A).

6. Key question 6: is it possible to complete with the 9-valent HPV vaccine for the women who are inoculated with the other HPV vaccine?

There have been no prior studies on whether the cross vaccination using 9-valent HPV vaccine is possible. Therefore, the decision was based on the recommendations of the Centers for Disease Control and Prevention. The Advisory Committee for Immunization Practice (ACIP) written by Petrosky et al. [21] recommends that if physician providing the vaccine does not know the type of previous vaccine, or if quadri- or bi-valent HPV vaccine are not well supplied, the vaccination schedule can be continued with 9-valent HPV vaccine in women vaccinated with quadri- or bi-valent HPV vaccine [21].

No recommendation level was established and the level of evidence was determined as $\mathrm{E}$ through the voting process of the committee because no studies have been conducted on this key question and the judgement was made on the basis of recommendations by ACIP.

- In cases of vaccinations started with other types of HPV vaccines, the 9-valent HPV vaccine may be used to continue or complete the vaccination ( ${ }^{\star}$ limited to cases where the previous vaccine types are unknown or unavailable) (E).

\section{Key question 7: is an additional 9-valent HPV vaccination possible to women who have already completed a series with another HPV vaccine product?}

Only 1 article for this key question was searched. Garland et al. [22] conducted a randomized, double-blind study to confirm safety and immunogenicity of additional vaccination using 9-valent HPV vaccine in 12-26 year old women who previously completed quadri-valent HPV vaccination. The 3 doses of 9-valent HPV vaccine were inoculated in 618 women of the study group, and 3 times of normal saline in 306 women of the control group. The efficacy of vaccination was confirmed by antibody titer. The antibody titer of HPV 6 , 11,16 , and 18 included in the quadri-valent vaccine were high for both group after 1st dose and as the vaccination progressed, antibody titer of 9 types of HPV were all increased in the study group compared to the control group. In addition, 98\% of 9-valent HPV vaccinated 
women were found to have antibodies to HPV $31,33,45,52$, and 58. Adverse events in vaccination site were found in $91 \%$ of the study group and $43.9 \%$ of the control group, and systemic side effects were observed similarly. Discontinuation of vaccination due to side effects was observed to be very low such as $0.5 \%$ for 9-valent $\mathrm{HPV}$ vaccine and $0 \%$ for control group, respectively.

For women over the age of 27 who have completed vaccination using quadri-valent vaccine, no current findings are available, but as identified in key question 3, 9-valent HPV vaccine is assumed to have the efficacy and safety. Therefore, the 9-valent vaccine can be inoculated to obtain additional prevention for HPV 31, 33, 43, 52, and 58 in consideration of clinical judgement and situation of subject. However, for women who have completed vaccination using bi-valent vaccine, it is not advisable to have an additional vaccination because there is no evidence for additional vaccination using 9-valent vaccine.

Since this article checked only immunogenicity in the age group which efficacy can be verified through cervical cytology or HPV test, the level of evidence was classified as B and the recommendation level was decided as weak via the voting process of committee.

- For women aged 12-26 years who have completed the quadri-valent HPV vaccination, an additional 9-valent HPV vaccination can be clinically determined considering the circumstances of the subject (2B).

\title{
DISCUSSION
}

The 9-valent HPV vaccine was found to be effective in preventing diseases associated with 9 types of $\operatorname{HPV}(6,11,16,18,31,33,45,52$, and 58$)$ by checking several clinical examination and antibody titer in 9-26 year old men and women. 9-valent HPV vaccine can be used in the middle-aged women with clinical judgment of individual risk factors and situations because the efficacy of 9-valent vaccine was confirmed by comparative trial with quadri-valent vaccine and the efficacy of quadri-valent vaccine was confirmed in the middle-aged women. In addition, the efficacy of 2 dose vaccination in 9-15 year old adolescents was confirmed by the geometrical mean titer compared with 3 dose vaccination in 16-26 year old women. If a vaccination is initiated with other vaccines, 9-valent HPV vaccine may be used to continue or complete the vaccination in cases of previously unknown or unavailable vaccines only. Since this recommendation is reviewed and written based on the articles published until 2016, it will have to be revised if the results of further studies on 9-valent HPV vaccine are published in the future.

\section{SUPPLEMENTARY MATERIALS}

\author{
Supplement 1 \\ Development process of 9-valent human papillomavirus (HPV) vaccine guideline
}

Click here to view 


\section{Supplement 2}

Evidence table and risk of bias

Click here to view

\section{REFERENCES}

1. Bauer HM, Ting Y, Greer CE, Chambers JC, Tashiro CJ, Chimera J, et al. Genital human papillomavirus infection in female university students as determined by a PCR-based method. JAMA 1991;265:472-7. PUBMED | CROSSREF

2. Schiffman M, Castle PE, Jeronimo J, Rodriguez AC, Wacholder S. Human papillomavirus and cervical cancer. Lancet 2007;370:890-907. PUBMED | CROSSREF

3. Konno R, Shin HR, Kim YT, Song YS, Sasagawa T, Inoue M, et al. Human papillomavirus infection and cervical cancer prevention in Japan and Korea. Vaccine 2008;26 Suppl 12:M30-42. PUBMED | CROSSREF

4. Ault KA; Future II Study Group. Effect of prophylactic human papillomavirus L1 virus-like-particle vaccine on risk of cervical intraepithelial neoplasia grade 2 , grade 3 , and adenocarcinoma in situ: a combined analysis of four randomised clinical trials. Lancet 2007;369:1861-8. PUBMED | CROSSREF

5. Gynecologic Cancer Prevention Committee. Clinical guideline of Cervarix®. Seoul: Korean Society of Gynecologic Oncology; 2011.

6. Gynecologic Cancer Prevention Committee. Clinical guideline of Gardasil@. Seoul: Korean Society of Gynecologic Oncology; 2011.

7. Min KJ, Kwon SH, Kim S, Kim HJ, Seong SJ, Song YJ, et al. Preventive vaccination against cervical cancer: Korean Society of Gynecologic Oncology Guideline. J Gynecol Oncol 2016;27:e30. PUBMED | CROSSREF

8. Luxembourg A, Brown D, Bouchard C, Giuliano AR, Iversen OE, Joura EA, et al. Phase II studies to select the formulation of a multivalent HPV L1 virus-like particle (VLP) vaccine. Hum Vaccin Immunother 2015;11:1313-22. PUBMED | CROSSREF

9. Joura EA, Giuliano AR, Iversen OE, Bouchard C, Mao C, Mehlsen J, et al. A 9-valent HPV vaccine against infection and intraepithelial neoplasia in women. N Engl J Med 2015;372:711-23. PUBMED | CROSSREF

10. Van Damme P, Olsson SE, Block S, Castellsague X, Gray GE, Herrera T, et al. Immunogenicity and safety of a 9-valent HPV vaccine. Pediatrics 2015;136:e28-39. PUBMED | CROSSREF

11. Castellsagué X, Giuliano AR, Goldstone S, Guevara A, Mogensen O, Palefsky JM, et al. Immunogenicity and safety of the 9-valent HPV vaccine in men. Vaccine 2015;33:6892-901. PUBMED | CROSSREF

12. Van Damme P, Meijer CJ, Kieninger D, Schuyleman A, Thomas S, Luxembourg A, et al. A phase III clinical study to compare the immunogenicity and safety of the 9-valent and quadrivalent HPV vaccines in men. Vaccine 2016;34:4205-12. PUBMED | CROSSREF

13. Muñoz N, Manalastas R Jr, Pitisuttithum P, Tresukosol D, Monsonego J, Ault K, et al. Safety, immunogenicity, and efficacy of quadrivalent human papillomavirus (types $6,11,16,18$ ) recombinant vaccine in women aged 24-45 years: a randomised, double-blind trial. Lancet 2009;373:1949-57. PUBMED | CROSSREF

14. Castellsagué X, Muñoz N, Pitisuttithum P, Ferris D, Monsonego J, Ault K, et al. End-of-study safety, immunogenicity, and efficacy of quadrivalent HPV (types $6,11,16,18$ ) recombinant vaccine in adult women 24-45 years of age. Br J Cancer 2011;105:28-37. PUBMED | CROSSREF

15. Luna J, Plata M, Gonzalez M, Correa A, Maldonado I, Nossa C, et al. Long-term follow-up observation of the safety, immunogenicity, and effectiveness of Gardasil ${ }^{\text {Im }}$ in adult women. PLoS One 2013;8:e83431. PUBMED | CROSSREF 
16. Iversen OE, Miranda MJ, Ulied A, Soerdal T, Lazarus E, Chokephaibulkit K, et al. Immunogenicity of the 9-valent HPV vaccine using 2-dose regimens in girls and boys vs a 3-dose regimen in women. JAMA 2016;316:2411-21. PUBMED | CROSSREF

17. Vesikari T, Brodszki N, van Damme P, Diez-Domingo J, Icardi G, Petersen LK, et al. A randomized, doubleblind, phase III study of the immunogenicity and safety of a 9-valent human papillomavirus L1 virus-like particle vaccine (V503) versus Gardasil@ in 9-15-year-old girls. Pediatr Infect Dis J 2015;34:992-8. PUBMED | CROSSREF

18. Moreira ED Jr, Block SL, Ferris D, Giuliano AR, Iversen OE, Joura EA, et al. Safety profile of the 9-valent HPV vaccine: a combined analysis of 7 phase III clinical trials. Pediatrics 2016;138:e20154387. PUBMED | CROSSREF

19. Schilling A, Parra MM, Gutierrez M, Restrepo J, Ucros S, Herrera T, et al. Coadministration of a 9-valent human papillomavirus vaccine with meningococcal and Tdap vaccines. Pediatrics 2015;136:e563-72. PUBMED | CROSSREF

20. Kosalaraksa P, Mehlsen J, Vesikari T, Forstén A, Helm K, Van Damme P, et al. An open-label, randomized study of a 9-valent human papillomavirus vaccine given concomitantly with diphtheria, tetanus, pertussis and poliomyelitis vaccines to healthy adolescents 11-15 years of age. Pediatr Infect Dis J 2015;34:627-34. PUBMED | CROSSREF

21. Petrosky E, Bocchini JA Jr, Hariri S, Chesson H, Curtis CR, Saraiya M, et al. Use of 9-valent human papillomavirus (HPV) vaccine: updated HPV vaccination recommendations of the advisory committee on immunization practices. MMWR Morb Mortal Wkly Rep 2015;64:300-4. PUBMED

22. Garland SM, Cheung TH, McNeill S, Petersen LK, Romaguera J, Vazquez-Narvaez J, et al. Safety and immunogenicity of a 9-valent HPV vaccine in females 12-26 years of age who previously received the quadrivalent HPV vaccine. Vaccine 2015;33:6855-64.

PUBMED | CROSSREF 